\title{
Hypertrophic Cardiomyopathy....in Search of its Own Identity
}

\author{
Jayesh V Trivedi* \\ Head of Department and Professor of Medicine, Gujarat Adani Institute of Medical Science, India
}

Received: September 12, 2017; Published: September 25, 2017

*Corresponding author: Jayesh V Trivedi, Head of Department and Professor of Medicine, Gujarat Adani Institute of Medical Science, Bhuj Gujarat, India, Email: drjvtrivedi@rediffmail.com

\section{Introduction}

Hypertrophic cardiomyopathy is a disease of muscle of heart. The incidence is $0.2 \%$ in general population. Only $15 \%$ of total patients are being diagnosed and large no of patients are yet to be screen-positive ,about $85 \%$.The motive of this article is to activate medical practioner to diagnose the disease early and prevent the complication ,one of them is sudden death. Incidence of sudden death is about $2 \%$ to $4 \%$.

\section{Etiology}

In $55 \%$ of patients it occurs in autosomal dominant conditions. It occurs due to mutation in one of the nine gene coding protein of cardiac sarcomere. B-MHC, Cardiac Troponin T, Myosin binding protein C, Alpha-Tropomyosin. This will result in cardiac Myocytes Hypertrophy, disturbances in alignment of muscles of heart and disruption of electrical functioning of heart. Its incidence is $33 \%$ in close relative of patient.

\section{Symptoms}

HCM can be asymptomatic. Or it may present as sudden death, syncopal attack, Dysponea or Angina. It is one of the commonest causes of sudden death in all cause mortality. The incidence is $2 \%$ to $4 \%$. Sudden death occurs in $30 \%$ of patients having HCM. The cause of sudden death is due to ventricular arrythmias, or left ventricular out flow tract obstruction. On clinical examination patient will have Bisferiens pulse, S4 gallop, Ejection systolic murmur at aortic area and murmur of Mitral regurgitation. ESM will be cresendo. Decresendo with normal aortic component os second heart sounds. There will be absence of Ejection systolic click.

\section{Diagnosis}

Electrocardiogram may show left ventricular hypertrophy, pathological Q wave due to septal hypertrophy and ventricular premature beats arising from left side of heart. Signs of biventricular failure are rarely seen. Presence of Atrial fibrillation is an ominous sign and it increases the cardiac mortality rate and decrease life expectency. Brocken brough Braunwald sign of failure of aortic pulse pressure to raise post PVC is a good marker in suspected patient.
Echocardiography is non invasive diagnostic tool to diagnose it correctly and to classify the HCM, in to Obstructive, Non obstructive or apical cardiomyopathy. Interventricular septal to left ventricular posterior wall thickness ratio of more than 1.33 is a good marker. Asymmetrical septal hypertrophy with left ventricular out flow tract obstruction will be seen. Left ventricular out flow gradient will be increased and it has a prognostic significance. Systolic Anterior Motion of anterior mitral leaflet with significant mitral regurgitation can be demonstrable [1-4].

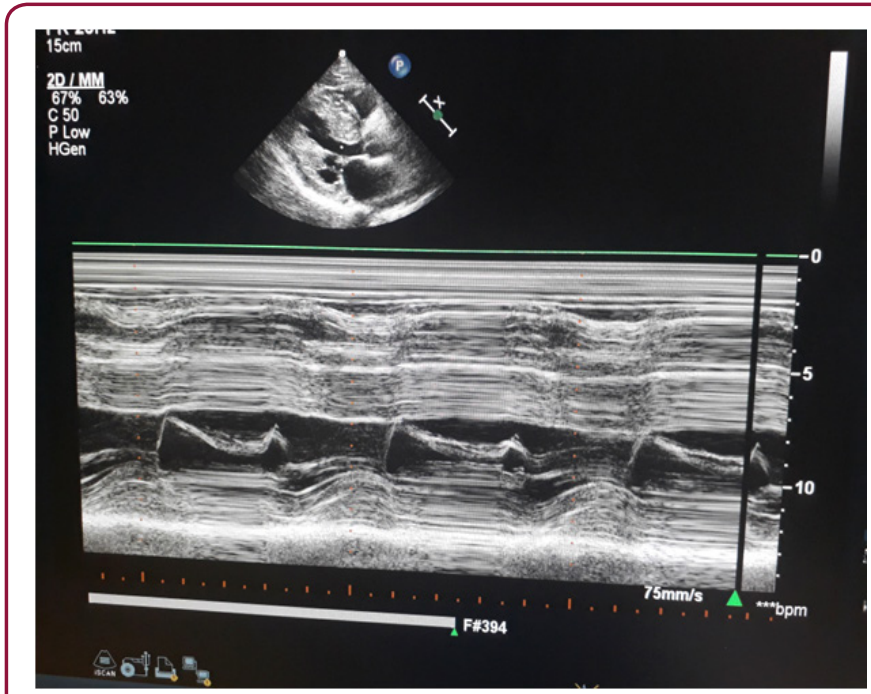

Figure 1 : Left Parasternal long axis view-m mode at mitral valve-thick IVS, IVS/ IVPE ratio more than 2, sam present.

Cardiac cath, Coronary angiography will be needed in some patients. Holter studies will be needed in patients in whom venticular arrythmias are to be rulled out. Implantable cardiac device, septoplasty are surgical treatment. Medical treatment will be beta blockers, verapamil or Diltiazem will be helpful. Digoxine or noradrnaline, imipramine group of drugs or diuretics are to be avoided. Amiodarone is a preferred anti arrhythmic drug and reduces the incidence of VT induced Sudden death. However 
intra cardiac defibrillator is preferred to medical therapy to treat Ventricular tachycardia. All family members of patient should be evaluated by echocardiogram to rule out asymptomatic HCM (Figures 1 \& 2).

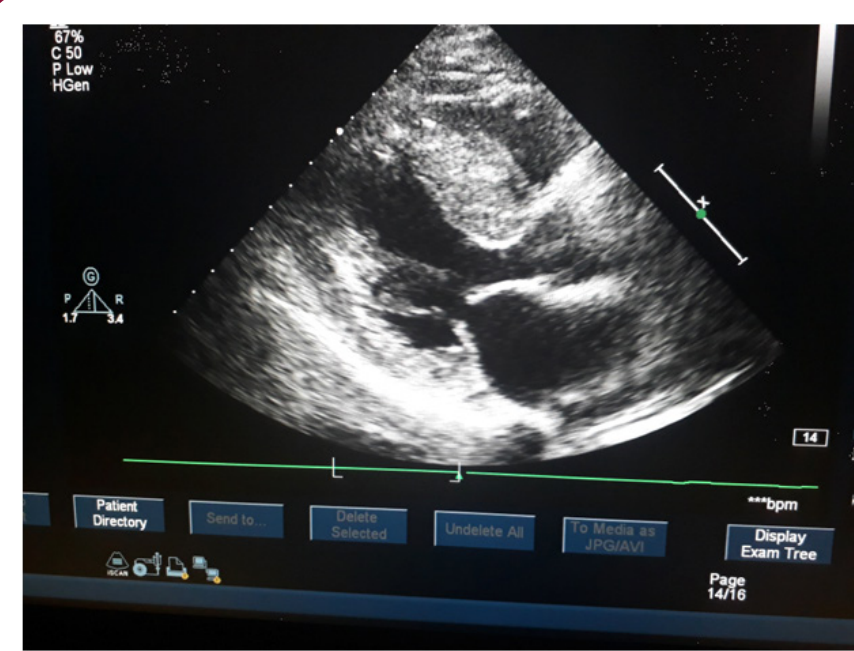

Figure 2 : Thick Ivs Obstructing Left Ventricular Out Flow Tract Partially.

\section{References}

1. Hypertrophic cardiomyopathy. Wikipedia, USA.

2. Hypertrophic Cardiomyopathy (2017) American Heart Association, Dallas, Texas, USA.

3. Hypertrophic cardiomyopathy (2017) British Heart Foundation, UK.

4. Overview (2017) Hypertrophic cardiomyopathy, Mayo Clinic, New York, USA.

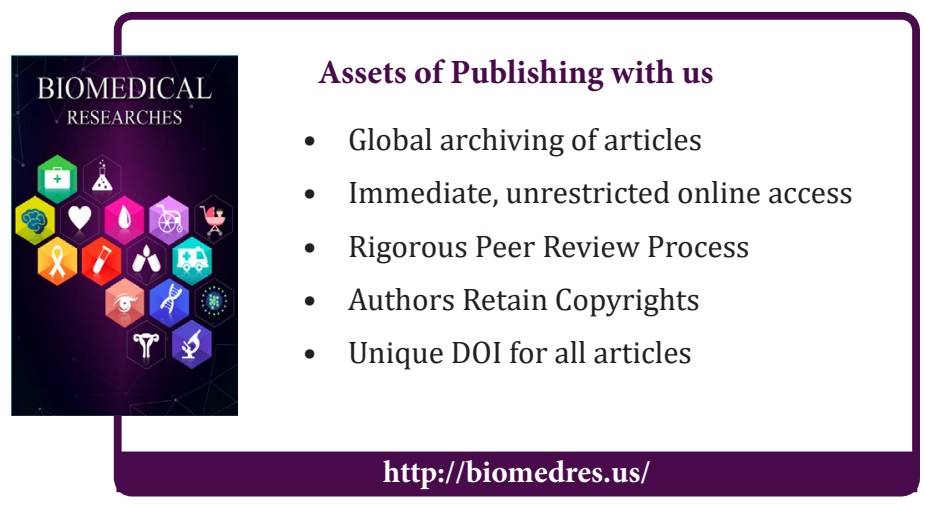

